\title{
La Incidencia de la Precipitación y el Área Sembrada Frente a la Producción de Arroz en el Departamento del Meta ${ }^{1}$
}

\section{The Incidence of Precipitation and the Area Sown Against Rice Production in the Department of Meta}

Ingrid del M. González P.*

Andrés F. Rodríguez C.***

José Isnardi Sastoque R.***

\section{Artículo de investigación \\ Recepción: 09/02/2019}

Aceptación: 12/14/2019

El Conuco es una revista de acceso abierto revisada por pares. (C) $2018 \mathrm{El}$ autor (es). Este es un artículo de acceso abierto distribuido bajo los términos de la Licencia Internacional Creative Commons Attribution 4.0 (CC-BY 4.0), que permite el uso, distribución y reproducción sin restricciones en cualquier medio, siempre que se acredite el autor y la fuente originales.

Consulte http://creativecommons.org/licenses/by/4.0/. ๑OPEN ACCESS

(c) $(9)$

* Código ORCID: https://orcid.org/0000-0001-6020-8338

** Código ORCID: https://orcid.org/0000-0003-4178-1117

*** Código ORCID: https://orcid.org/0000-0002-0502-9879

Como citar este artículo / To reference this articcle: R. (2016). La Incidencia de la Precipitación y el Área

CO: (investigación, economia y sociedad), 3(1), pp. 1-15.
DOI: https://doi.org/10.22579/2619-614X.532

\section{Resumen}

El objetivo de este artículo es analizar mediante el método de regresión lineal simple, la relación del impacto que tiene la precipitación y la cantidad de área sembrada frente a la producción de arroz en el Departamento del Meta. Para este análisis se obtuvieron datos de precipitación proporcionados por el IDEAM y datos de área sembrada y producción de arroz obtenida de la información anexada del IV Censo Nacional Arrocero del año 2016, realizado por el convenio FEDEARROZ-DANE. Los valores que se analizaron de estas tres variables fueron de los períodos semestrales comprendidos entre (1999 - 2017). Para el caso de la precipitación se halló el promedio usando las mediciones totales semestrales de las estaciones pluviométricas ubicadas en el departamento del Meta. Después del análisis, se obtuvo como resultados que la producción de arroz es una variable dependiente de la cantidad de área que se siembre y de la cuota pluviométrica como valor agregado, es decir, a mayor cantidad de área sembrada y una buena pluviosidad en el ciclo productivo, mayor será la producción de arroz.

Palabras Clave. Precipitación pluviométrica, producción de arroz, área siembra, regresión, incidencia

Códigos JEL: Q1, Q14, Q54

1 Artículo derivado del proyecto de investigación: "Análisis de las condiciones de producción y comercialización del sector arrocero en el departamento del Meta" 


\section{Abstract}

The objective of this article is to analyze the relationship of the impact of precipitation and the amount of area sown against rice production in the Meta department. For this analysis were obtained precipitation data provided by the IDEAM and data of sown area and production of rice obtained from the information annexed to the IV national census rice of the year 2016, carried out by the Convention FEDEARROZ-DANE. The values that were analyzed from these three variables were from the semiannual periods between (1999 -2017). In the case of precipitation, the average was found using the total semester measurements of the Pluviometric stations located at the target. After the analysis, it was obtained as results that the production of rice is a variable dependent on the amount of area that is sown and of the quota Pluviometric as value added, ie, the greater amount of sown area and a good rainfall in the cycle Productive, the greater the rice production will be.

Keywords. Pluviometric precipitation, rice production, planting area, regression, incidence

\section{Introducción}

El arroz, el trigo y el maíz son los tres cultivos alimentarios más importantes del mundo; juntos suministran directamente más del $50 \%$ de todas las calorías consumidas por la población humana (Gnanamanickam, 2009). De los cereales antes mencionados, el trigo es el líder en el área cosechada, cada año con 214 millones de hectáreas, seguido del arroz con 154 millones de hectáreas y el maíz con 140 millones de hectáreas (Kennedy, 2002), El arroz proporciona el 21\% de la energía per cápita humana global y el 15\% de la proteína per cápita (FAO 2017)

Además, el consumo mundial promedio de arroz en 1999 fue de $58 \mathrm{~kg}$, siendo el consumo más alto en algunos países asiáticos; Myanmar tiene el mayor consumo anual con $211 \mathrm{~kg} /$ persona. Los que comen arroz y los cultivadores constituyen la mayor parte de los pobres del mundo: según el Informe de Desarrollo Humano del PNUD para 1997, aproximadamente el $70 \%$ de los $1.300 \mathrm{mi}-$ llones de pobres del mundo viven en Asia, donde el arroz es el alimento básico (FAO, 2004)

En Colombia se cultiva arroz en cinco zonas que han sido definidas por el gremio arrocero y específicamente en la zona Llanos de la cual hace parte el departamento del Meta existen municipios con una gran cantidad de hectáreas sembradas, cuyos cultivadores se ven expuestos a riesgos por diferentes variables como la precipitación, la temperatura y el brillo solar. Por lo tanto, este estudio buscó determinar la incidencia de la precipitación y el tamaño del área sembrada frente a la producción en toneladas, como parte del proyecto macro que busca determinar un instrumento financiero derivado que sirva de cobertura frente a cambios climáticos que perjudiquen al productor.

Para el desarrollo del trabajo se acudió a fuentes primarias realizando entrevistas a cultivadores del cereal y al gremio arrocero del departamento; secundarias como la literatura existente en libros y revistas y a la aplicación de técnicas estadísticas en la aplicacion informática Excel, cuyo objetivo pretendía establecer como las variables independientes (precipitación y área sembrada) afectaban la variable dependiente (producción) en un periodo de tiempo, según los datos suministrados por el IDEAM y FEDEARROZ.

En la literatura consultada se encontró que las variables precipitación, temperatura, y radiación solar afectan directamente los procesos fisiológicos de la planta de arroz, que de una u otra manera inciden en la producción de grano e in- 
directamente inciden en la presencia de plagas y enfermedades del cultivo. (Sativa, 2003)

Además, el arroz requiere más agua que cualquier otro cultivo. Como resultado, el cultivo de arroz solo se realiza en aquellas áreas donde la precipitación mínima es de $115 \mathrm{~cm}$. Aunque las regiones están teniendo un promedio anual de lluvia entre 175 a $300 \mathrm{~cm}$, son las más adecuadas. También necesita condiciones de inundación con la profundidad del agua que varía más de $25 \mathrm{~mm}$, en el momento del trasplante hasta $150 \mathrm{~mm}$, durante 10 semanas del período de crecimiento. (SAG, 2010) (Secretaría de Agricultura y Ganadería, SAG, 2010)

\section{Materiales y Métodos}

Se realizará un análisis sobre el impacto que generan la cantidad de área que se siembra junto a las precipitaciones en el cultivo de arroz, para conocer de qué manera afecta a la producción de este cereal. Para el desarrollo de esta fase se utilizaran técnicas estadísticas como lo es el método de regresión lineal (cuyas variables independientes son los datos obtenidos del área sembrada y de la precipitación media y la variable dependiente que para este caso es la producción de arroz en el Departamento del Meta en los periodos comprendidos entre los años 1999 al 2017 por semestre), que conlleva a la aplicación de metodologías como el método de mínimos cuadrados perfectos y el coeficiente de determinación; el cual nos permitirá conocer el grado de influencia de estas variables mencionadas anteriormente.

El área de estudio es el Departamento del Meta ubicado en la región Orinoquia de Colombia ubicado geográficamente en la parte central del mismo como se muestra en la figura 1.

Figura 1. Ubicación geográfica nacional de los Ilanos orientales Fuente: Google Maps

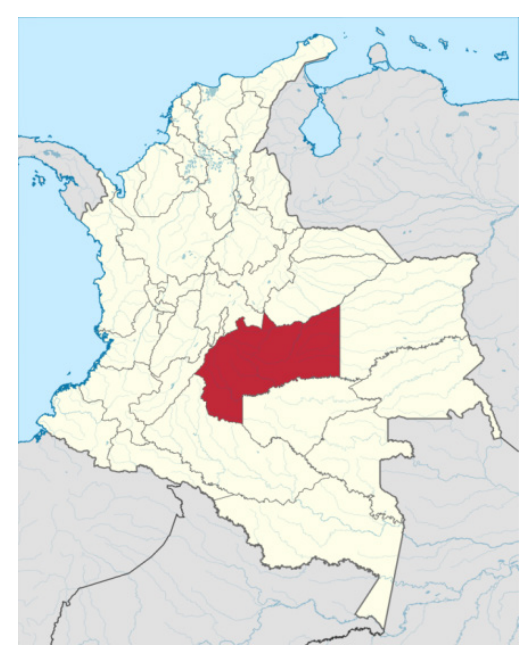

\section{Análisis teórico del método de regresión lineal simple y la producción de arroz en el departamento de Meta}

\section{Método de regresión lineal simple.}

La producción de arroz con respecto a los diferentes factores que influyen ya sea positiva o negativamente se puede conocer partiendo del método estadístico de regresión lineal, pues con este se puede saber qué tanto influyen esos factores externos que se consideran variables independientes, frente a la producción denominada variable dependiente.

Este modelo se realiza mediante un diagrama de dispersión, el cual refleja la relación entre la variable dependiente en el cual se ubica en el eje vertical (Y), y la variable independiente en donde está ubicada en el eje horizontal (X), de acuerdo a (Universidad Carlos III de Madrid, 2000)

Figura 2 Diagrama de dispersión 


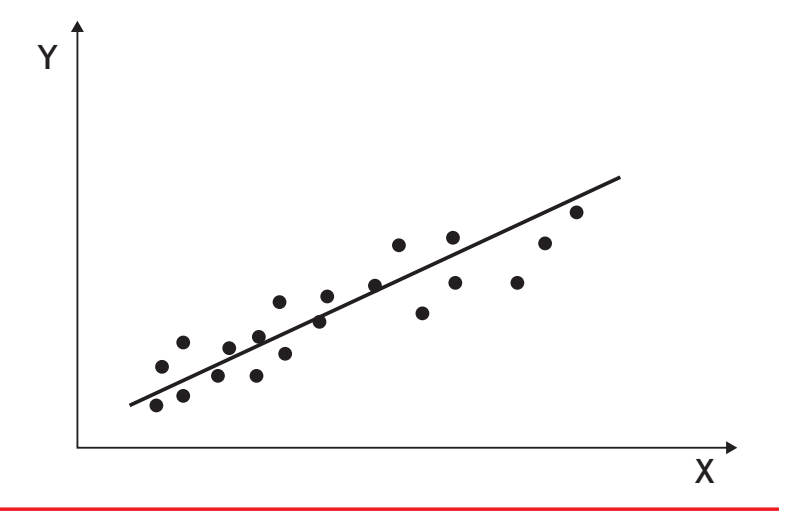

Para determinar la relación entre que refleja en el diagrama de dispersión mediante la línea de tendencia la cual es recta (de ahí el nombre de regresión lineal), se realiza una ecuación de mejor ajuste la cual es.

\section{Producción de arroz en el departamento de Meta.}

La producción de arroz en el departamento del Meta, de acuerdo a la información reportada por el DANE, y que se muestra en la tabla 1 y figura 2, se aprecia la producción real en el departamento de los últimos cinco años del último censo arrocero nacional (Federación Nacional de Arroceros, Fedearroz, 2012)

En el departamento del Meta, la mayor producción arrocera fue en el año 2013 con una producción del 82.672 Toneladas, que corresponde a un $28,5 \%$ del total de la producción del semestre I de los últimos cinco años del cuarto censo na- cional arrocero; que frente a los siguientes años el comportamiento productivo, en el 2014 disminuyo significativamente en 40.806 toneladas, correspondiente a un grado porcentual de $44,4 \%$, sin embargo para el año 2015 la producción volvió a incrementar en un 17,6\%, que corresponde a 7.386 toneladas.

No obstante, para el segundo semestre de los últimos cinco años del cuarto censo arrocero, el comportamiento productivo del arroces en el departamento del meta, se mostró, que el año de mayo producción de arroz fue el año 2016 con una producción de 343.717 Toneladas de arroz, sin embargo la menor producción para este semestre fue en el año 2014 con una producción de arroz de 150.769 Toneladas de arroz, resaltando que para el año 2012 la producción fue alta en 275.707 toneladas de arroz y para los años 2013 y 2014 disminuyeron significativamente (Fedearroz, 2017).

De los semestres de producción de arroz en el meta, de acuerdo a la figura No 3, la mayor productividad que obtuvo el departamento fue en el semestre 2; Sin embargo, el año de mayor productividad fue el año 2016, seguido del año 2012

El cambio climático indiscutiblemente tiene un impacto significativo en el sector agrícola en Colombia, que representa más de una décima parte del PIB del país y emplea a más de una quinta parte de su población (Ocampo, 2012), .

Tabla 1: Producción del Arroz en el Meta por Semestre

\begin{tabular}{ccccccc}
\hline \multirow{2}{*}{ Variable } & \multicolumn{7}{c}{ Producción del arroz en el departamento del meta } \\
\cline { 2 - 7 } & $\mathbf{2 0 1 2 .}$ & $\mathbf{2 0 1 3 .}$ & $\mathbf{2 0 1 4}$ & $\mathbf{2 0 1 5 .}$ & $\mathbf{2 0 1 6 .}$ & Total \\
\hline Semestre-1. & 50.960 & 82.672 & 41.866 & 49.252 & 65.518 & 290.269 \\
\hline Semestre-2. & 275.707 & 224.181 & 150.769 & 234.395 & 343.717 & 1.228 .769 \\
\hline Total & $\mathbf{3 2 6 . 6 6 7}$ & $\mathbf{3 0 6 . 8 5 3}$ & $\mathbf{1 9 2 . 6 3 5}$ & $\mathbf{2 8 3 . 6 4 8}$ & $\mathbf{4 0 9 . 2 3 5}$ \\
\hline
\end{tabular}

Fuente: Elaboración propia, datos tomados del $4^{\circ}$ censo nacional arrocero del 2016 publicado en la página del DANE. 


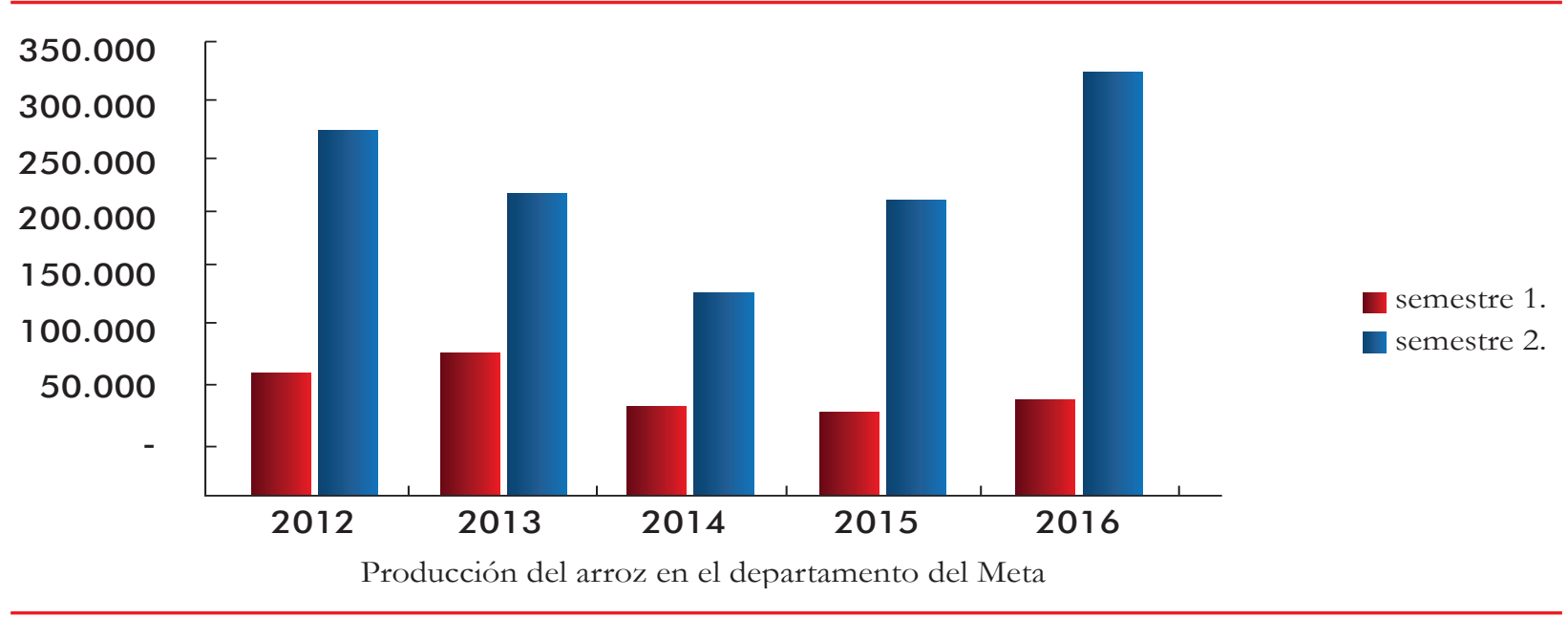

Los análisis indican que para el año 2050, probablemente habrá un aumento significativo de la temperatura, una precipitación más errática y una mayor prevalencia de plagas y enfermedades. Para abordar las extensas implicaciones socioeconómicas de estos efectos, el gobierno debe priorizar la adaptación, invirtiendo en evaluaciones regionales; investigación y desarrollo; y transferencia de tecnología y capacitación para agricultores. (Pabón, 2012)

\section{Últimas décadas y efectos ambientales en la agricultura.}

En la última década, las variaciones climáticas relacionadas con El Niño y La Niña han planteado serios desafíos para la agricultura colombiana, lo que demuestra que muchos agricultores no pueden manejar eficazmente el riesgo y adaptarse a las fluctuaciones y choques climáticos. El cambio climático antropogénico es probable que exacerbe esta situación. Los científicos proyectan aumentos en la variabilidad del clima, temperaturas más altas y precipitaciones erráticas (Ocampo 2012).

En Colombia, el aumento medio esperado en la temperatura media anual para 2050 se estima en $2.5^{\circ} \mathrm{C}$, y es probable que las precipitaciones aumenten en un $2.5 \%$ para mediados de siglo. En efecto, sin adaptación acelerada,

- Degradación del suelo y pérdidas de materia orgánica en las laderas de los Andes.

- Inundaciones en las costas del Caribe y Pacífico.

- Pérdidas de nicho para café, frutas, cacao y bananas.

- Cambios en la prevalencia de plagas y enfermedades.

- Fusión de glaciares y estrés hídrico. (Ocampo, 2012)

Las proyecciones muestran que, para 2050, el $80 \%$ de los cultivos probablemente se verá afectado en la mayoría (> 60\%) de sus actuales áreas de cultivo, con impactos particularmente graves en los cultivos perennes de alto valor. Estas proyecciones tienen importantes implicaciones socioeconómicas: el sector agrícola representa el $40 \%$ de las exportaciones colombianas y el 21\% de la población depende directamente de la agri- 
cultura para el empleo. Por lo tanto, el cambio climático tendría efectos de gran alcance en las agroindustrias, las cadenas de suministro y la seguridad alimentaria y nutricional. (Pabón, 2012)

Además, los efectos de los cambios climáticos afectan especialmente a los productores de pequeña escala y de escasos recursos. Los pequeños agricultores representan la gran mayoría de los productores de alimentos en Colombia: para los cultivos de exportación (que generan el 41\% del PIB agrícola), solo la caña de azúcar se cultiva principalmente en granjas grandes; y para los cereales, café, cacao y Musa (por ejemplo, banano y plátano), el $50 \%-90 \%$ de los productores son pequeños agricultores, con un tamaño de finca de menos de 10 hectáreas cada uno. (Agricultura Colombiana: Adaptación al Cambio Climático, 2017) Los autores son: Charlotte Lau, Andy Jarvis y Julián Ramírez, es un documento del Centro de Investigación de Agricultura Tropical, CIAT.

\section{Precipitación errática.}

Es probable que los períodos más secos a lo largo del año sean menos secos, mientras que se prevé que los períodos más húmedos se vuelvan más húmedos. Las predicciones muestran un aumento promedio de $2.5 \%$ a nivel nacional para 2050, con un cambio mínimo de $-1.4 \%$ en Cesar y un máximo de $5.6 \%$ en Huila. Las únicas excepciones son las regiones secas en la costa del Caribe, cuyas probables disminuciones en las precipitaciones constituyen una causa importante de preocupación. (Precipitación errática Colombia)

Los científicos pronostican que el 36\% de los cultivos enfrentará una precipitación superior al $3 \%$ en al menos el $60 \%$ de las áreas en las que se cultivan. Los cambios en los patrones de precipitación pueden alterar las fechas de floración; afectan los factores bióticos (por ejemplo, plagas, enfermedades, malezas) en diferentes sistemas de producción, lo que aumenta los costos de producción; y cambiar la disponibilidad de agua del suelo. Las fuertes lluvias pueden provocar inundaciones, erosión del suelo y pérdidas masivas de cultivos. Esto puede ser exacerbado en la costa del Pacífico, donde el aumento del nivel del mar también puede causar inundaciones y salinización de los suelos.

\section{El arroz en Colombia tras los fenómenos climáticos.}

Las líneas de arroz también se están evaluando para otros rasgos, como la arquitectura de la planta, el tipo de grano y la resistencia a los estreses bióticos y abióticos. En particular, los científicos han utilizado la investigación sobre reproducción y patología para desarrollar cultivos con resistencia duradera a las plagas y enfermedades. Esta tarea a menudo se complica por la diversidad entre las muchas variaciones de algunos patógenos, que les permiten mutar, diversificar y duplicar rápidamente y dificultar el desarrollo de cultivos con resistencia adecuada.

Entre 1967 y 2005, Colombia ha lanzado 41 variedades diferentes de arroz, que han generado beneficios económicos estimados en cientos de millones de dólares estadounidenses, debido al aumento de los rendimientos y la reducción de los gastos en pesticidas. Las variedades recientemente desarrolladas son resistentes a la plaga de sogata, la hoja blanca y la explosión del arroz, tres de las plagas y enfermedades más destructivas del cultivo de arroz.

\section{Resultados}

Como se puede observar en la tabla No 1 una vez analizados los periodos comprendidos entre 1999-2017, se puede evidenciar que hubo varia- 
ciones en la cantidad de área sembrada, donde se muestra que en el primer semestre de cada año es el periodo en donde los agricultores siembran más el arroz, esto significa que para el segundo semestre se recoge la producción de arroz y paralelamente se refleja la mayor producción de este para el segundo periodo; pueden que algunos arroceros siembren el cultivo en segundo semestre para poder recolectar a principios del siguiente año.

En la tabla 2, se reflejan los datos proporcionados por el análisis de regresión realizado con los datos de área sembrada y producción. Los datos muestran que hay una relación entre la producción respecto al área sembrada de un 64,84\% reflejado en el coeficiente de determinación $\left(\mathrm{R}^{\wedge} 2\right)$. También se muestran los valores de intercepción y de la variable con los cuales se pueden calcular unos estimados de producciones futuras.

La figura No 4 ilustra los datos que contiene la tabla No 2, donde muestra una línea de tendencia que expresa que los resultados tienden a una baja en área sembrada en años futuros, lo que significa que afectaría de manera significativa la producción de arroz. No obstante, para comprobar los resultados, a continuación, se muestra en la figura 4 la ecuación con la cual se pueden hallar producciones futuras con los valores reflejados en la tabla N. 3 encontrados en la intercepción y la variable $\mathrm{X} 1$.
Tabla 2: Relación Producción/ Área Sembrada

\begin{tabular}{|c|c|c|}
\hline Año & Produccion & A Sembrada \\
\hline $1999-1$ & $102,578 \mathrm{t}$ & 87,204 ha \\
\hline $1999-2$ & $484,578 \mathrm{t}$ & 18,183 ha \\
\hline $2000-1$ & $99,412 \mathrm{t}$ & 84,787 ha \\
\hline $2000-2$ & $449,407 \mathrm{t}$ & 14,111 ha \\
\hline $2001-1$ & $76,409 \mathrm{t}$ & 77,386 ha \\
\hline $2001-2$ & $424,627 \mathrm{t}$ & 18,239 ha \\
\hline $2002-1$ & $102,975 \mathrm{t}$ & 71,524 ha \\
\hline $2002-2$ & $387,528 \mathrm{t}$ & 13,981 ha \\
\hline 2003-1 & $79,283 \mathrm{t}$ & 82,615 ha \\
\hline 2003-2 & $466,038 \mathrm{t}$ & 17,003 ha \\
\hline $2004-1$ & $89,855 \mathrm{t}$ & 83,848 ha \\
\hline 2004-2 & $460,311 \mathrm{t}$ & 17,789 ha \\
\hline $2005-1$ & $89,428 \mathrm{t}$ & 71,025 ha \\
\hline $2005-2$ & $378,146 \mathrm{t}$ & 11,401 ha \\
\hline $2006-1$ & $61,819 \mathrm{t}$ & 53,051 ha \\
\hline $2006-2$ & $291,007 \mathrm{t}$ & 10,723 ha \\
\hline $2007-1$ & $60,872 \mathrm{t}$ & 59,249 ha \\
\hline $2007-2$ & $335,181 \mathrm{t}$ & 14,756 ha \\
\hline $2008-1$ & $86,487 \mathrm{t}$ & 72,257 ha \\
\hline $2008-2$ & $415,349 \mathrm{t}$ & 11,546 ha \\
\hline $2009-1$ & $62,695 \mathrm{t}$ & 96,057 ha \\
\hline $2009-2$ & $504,863 \mathrm{t}$ & 7,894 ha \\
\hline $2010-1$ & $42,503 \mathrm{t}$ & 80,700 ha \\
\hline $2010-2$ & $447,577 \mathrm{t}$ & 16,481 ha \\
\hline $2011-1$ & $85,081 \mathrm{t}$ & 86,983 ha \\
\hline $2011-2$ & $306,385 \mathrm{t}$ & 10,752 ha \\
\hline $2012-1$ & $50,960 \mathrm{t}$ & 56,719 ha \\
\hline $2012-2$ & $275,707 \mathrm{t}$ & 16,383 ha \\
\hline 2013-1 & $82,672 \mathrm{t}$ & 61,640 ha \\
\hline 2013-2 & $224,181 \mathrm{t}$ & 8,655 ha \\
\hline $2014-1$ & $41,866 \mathrm{t}$ & 31,083 ha \\
\hline $2014-2$ & $150,769 \mathrm{t}$ & 8,680 ha \\
\hline $2015-1$ & $49,252 \mathrm{t}$ & 47,263 ha \\
\hline $2015-2$ & $234,395 \mathrm{t}$ & 16,258 ha \\
\hline 2016-1 & $65,518 \mathrm{t}$ & 63,312 ha \\
\hline 2016-2 & $343,717 \mathrm{t}$ & 14,024 ha \\
\hline $2017-1$ & $74,395 \mathrm{t}$ & 68,052 ha \\
\hline $2017-2$ & $319,637 \mathrm{t}$ & $14,705 \mathrm{ha}$ \\
\hline
\end{tabular}

Fuente: Propia, elaborado a partir de datos de la FEDEARROZ

Tabla 3: Estadísticas de Regresión Producción Vs Área Sembrada

\begin{tabular}{|c|c|c|c|c|c|c|c|}
\hline \multicolumn{8}{|c|}{ Estadísticas de la regresión } \\
\hline & & \multicolumn{3}{|c|}{ Coeficiente de correlación múltiple } & 0.80529423 & & \\
\hline & & \multicolumn{3}{|c|}{ Coeficiente de determinación $\mathrm{R}^{\wedge} 2$} & 0.6484988 & & \\
\hline & & \multicolumn{3}{|c|}{$\mathrm{R}^{\wedge} 2$ ajustado } & 0.63873488 & & \\
\hline & & \multicolumn{3}{|c|}{ Error típico } & 97741.5558 & & \\
\hline & & \multicolumn{3}{|c|}{ Observaciones } & \multicolumn{2}{|l|}{38} & \\
\hline & Coeficientes & Error típico & Estadístico t & Probabilidad & Inferior $95 \%$ & Superior $95 \%$ & Superior $95.0 \%$ \\
\hline Intercepción & 396737.674 & 27012.1331 & 14.6873878 & $8.8991 \mathrm{E}-17$ & 341954.529 & 451520.819 & 451520.819 \\
\hline Variable X 1 & -4.24261605 & 0.52058458 & -8.14971523 & $1.0852 \mathrm{E}-09$ & -5.2984105 & -3.18682159 & -3.18682159 \\
\hline
\end{tabular}


Figura 4 Producción Vs Área Sembrada

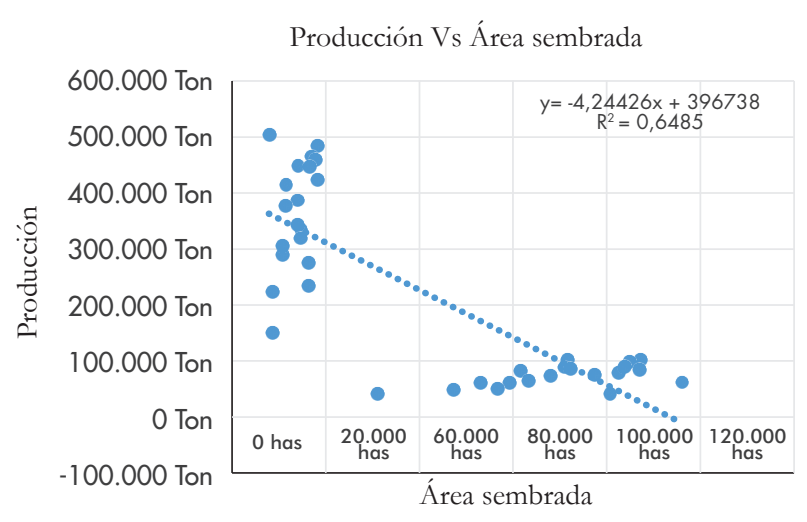

La tabla $\mathrm{N}^{\circ} 5$ (Ver apéndice A) muestra tanto el total de la precipitación en medidas mms de los años 1999-2017, como la productividad del arroz durante los mismos periodos medida en t. Reflejando las proporciones de producción y precipitación a lo largo del periodo, donde se muestra una variación muy constante en el promedio de lluvias que se dieron en el departamento del Meta.

En la tabla No 6 (Ver apéndice B) el análisis de regresión arroja unos datos que demuestran la incidencia de la precipitación frente a la producción.
Se observa que la relación de la precipitación hacia la producción no es muy alta, pues para que esta pueda influir en un gran porcentaje se necesitan de otras variables que influyan de manera significativa la producción de arroz. La figura 5 refleja los datos de la tabla $\mathrm{N}^{0} 3$ cuya línea de tendencia muestra una leve baja, también se encuentra la ecuación cuyos valores se utilizarán para proyectar futuras producciones.

De acuerdo a la tabla No 8 (Ver apéndice C) se evidencia la relación que tienen la cantidad de área sembrada y el nivel de precipitación sobre la producción de arroz, generando un impacto positivo para la productividad del sector arrocero. La tabla 6 (Ver apéndice D) que es el análisis de regresión, demuestra que existe relación entre la cantidad de área sembrada, la precipitación y la producción de arroz con porcentaje de exactitud de $65.24 \%$, lo cual indica que a mayor cantidad de hectáreas sembradas con buen índice de lluvias, mayor producción habrá.

\section{Conclusiones}

El artículo es el resultado final y el valor agregado como aporte al conocimiento generado por el de-

Figura 5 Producción Vs Precipitación

Producción Vs Precipitación

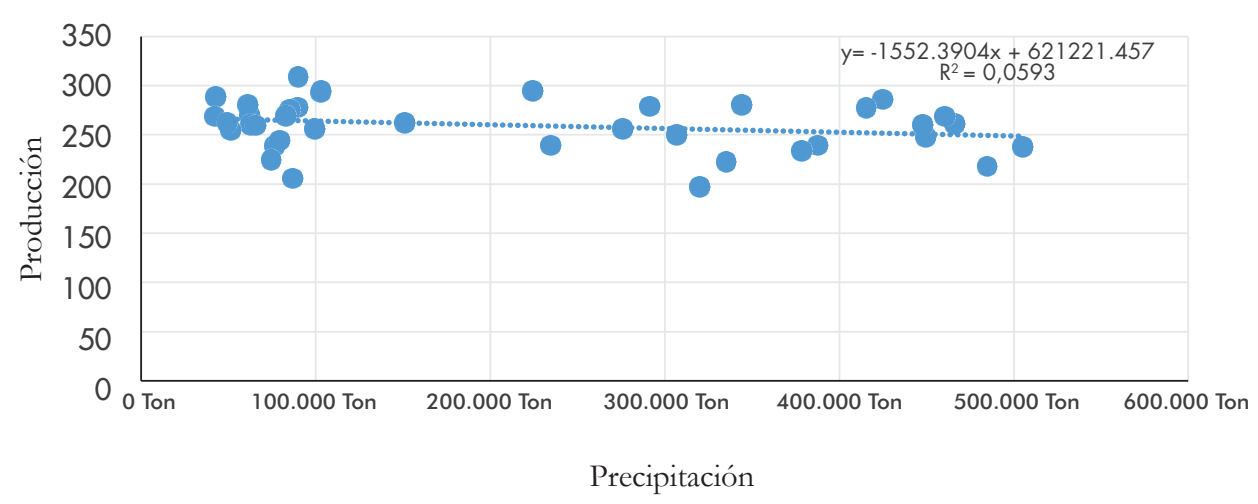


sarrollo de la investigación sobre la incidencia de la precipitación y el área sembrada frente a la producción de arroz en el Departamento del Meta:

El análisis de regresión encontrado en la tabla 6, muestra unos valores denominados intercepción con valor de 499.969.47, variable x 1 con valor de -410.38, y variable x 2 con valor de -4.1657 . Estos valores son necesarios para hallar las futuras producciones que se podrían dar en el Departamento del Meta teniendo en cuenta un estimado de área sembrada y un estimado de un promedio total de precipitaciones.

Se estima que para el 2018, en el primer semestre habrá un total de área sembrada de 70.000 hay un promedio de precipitación de $236 \mathrm{~mm}$,

\begin{tabular}{ccc}
\hline Año & Precipitación & A. Sembrada \\
\hline $2018-1$ & $236 \mathrm{~mm}$, & $70,000 \mathrm{ha}$ \\
\hline $2018-2$ & $186 \mathrm{~mm}$, & $12,000 \mathrm{ha}$ \\
\hline $2019-1$ & $180 \mathrm{~mm}$, & $80,000 \mathrm{ha}$ \\
\hline
\end{tabular}

Para hallar la proyección, se realiza la ecuación:

Donde a es el valor de la intercepción, b1 es el valor de la variable x1, x1 es el valor de la precipitación estimada, b2 es el valor de la variable $\mathrm{x} 2, \mathrm{y}$ x2 es el valor estimado del área sembrada.

$\mathrm{Al}$ asignar los valores respectivos en la ecuación, queda así:
Para el 2018-1:

Para el 2018-2:

Para el 2019-1

$\mathrm{Al}$ solucionar las ecuaciones anteriores, se tienen los resultados en la siguiente tabla:

\begin{tabular}{cccc}
\hline $2018-1$ & $111,513 \mathrm{t}$ & 236 & 70,000 ha \\
\hline $2018-2$ & $373,648 \mathrm{t}$ & 186 & 12,000 ha \\
\hline $2019-1$ & $92,837 \mathrm{t}$ & 180 & 80,000 ha \\
\hline
\end{tabular}

Como se puede evidenciar, tanto en la práctica como en la teoría, la cantidad de área sembrada y la precipitación son esenciales para producción de arroz, pues como se observa en la anterior tabla, entre más suba la cantidad de área sembrada, pero sin un buen nivel de precipitación, la producción puede bajar lo cual puede ser perjudicial para el productor arrocero, pero si el nivel de lluvia va en conjunto con la cantidad sembrada, la cantidad de producción será mayor. Sin embargo, este artículo se aleja de las dificultas que representa para la industria arrocera una precipitación excesiva en relación al levantamiento de la cosecha de este cereal. Se puede decir que, si bien las precipitaciones son vitales para la germinación de la semilla y el florecimiento del grano comestible, sin embargo, las mismas lluvias se ponen en contra en el momento de la cosecha. 


\section{Referencias}

Lau, C.; Jarvis, A.; Ramírez, J. (2011) Agricultura Colombiana: Adaptación al Cambio Climático. CIAT Politicas en Sintesis no. 1 Centro Internacional de Agricultura Tropical (CIAT) Cali - Colombia. 4 p. Recuperado de: https://cgspace.cgiar.org/handle/10568/57475 https://hdl.handle.net/10568/57475

El Tiempo . (2005). Meta, Primero en Palma, Soya y Arro\%. Edición del 29-06-2005 Recuperado el 2018, de El Tiempo : https://www.eltiempo.com/archivo/ documento/MAM-1635388

FAO. (2004). El Arroz y la Nutrición Humana . Recuperado el 2018, de fao.org: http://www.fao.org/rice2004/es/fsheet/hoja3.pdf

FAO. (2017). El futuro de la alimentación y la agricultura . Obtenido de Organización de las Naciones Unidas para la Alimentación y la Agricultura: http://www. fao.org/3/a-i6881s.pdf

Fedearroz. (2012). Dinamica del Sector Arrocero de los Llanos Orientales de Colombia. Recuperado de: http://www.fedearroz. com.co/doc_economia/Dinamica_ del_sector_arrocero_en_los_Llanos_ orientales.pdf

Fedearroz. (2015). Colombia unida frente a un gran desastre. Recuperado el 2018, de Fedearroz: http://www.fedearroz. com.co/revistanew/arroz489.pdf
Fedearroz. (2017). Historia del Arroz: Recuperado el 2018, de Fedearroz: http:// www.fedearroz.com.co/new/historiaarroz.php

Gnanamanickam, S. (2009). El arroz y su importancia para la vida bumana. Obtenido de Springer: https://link.springer.com/chapter/10.1007/978-90-481-2465-7_1

kennedy, D. (2002). The Importance of Rice. Science 05 Apr 2002. Vol. 296, Issue 5565, pp 13 DOI: 10.1126/ science.296.5565.13 Obtenido de: http://science.sciencemag.org/content/296/5565/13

Ocampo, O. (2012). El cambio climático y su impacto en el agro. Revista de Ingenieria No. 33, enero - junio, 2011, pp 115-123 ISSN: 0121-4993 Universidad de los Andes - Colombia. Obtenido de: https://www.redalyc.org/ pdf/1210/121022658012.pdf

Pabón, J. (2012). Cambio Climatico en Colombia: Tendencias en la Segunda Mitad del Siglo XX y Escenarios Posibles para el Siglo XXI. Volumen XXXVI, Número 139 Junio 2012 Obtenido de: http://www.scielo. org.co/scielo.php?script=sci_arttext\& pid $=$ S0370-39082012000200010

Sag. (2010). Manual Técnico para el Cultivo del Arro: Recuperado de: SAG: https://curlacavunah.files.wordpress. com/2010/04/el-cultivo-del-arroz.pdf 


\section{Referencias}

Sativa, O. (2003). Manual Técnico para el Cultivo del Arroz: Obtenido de: Secretaria de Agricultura y Ganadería (SAG): https://curlacavunah.files.wordpress. com/2010/04/el-cultivo-del-arroz.pdf

Senara. (2010). Tecnología de producción para el cultivo de arroz en riego. Recuperado el 2018, de Convenio Mag-Senara-IICA: http://www.mag.go.cr/bibliotecavirtual/AV-1389.pdf
UCM. (2018). Los alimentos como fuente de energia, nutrientes y otros bioactivos. Obtenido de Manual de Nutrición y Dietética: https://www.ucm.es/data/cont/ docs /458-2018-01-10-cap-14-alimentos-2018.pdf

Universidad Carlos III de Madrid. (2000). Análisis de Regresión Lineal, el Procedimiento Regresión Lineal. Obtenido de Universidad Carlos III de Madrid: https://www.uc3m.es/ss/Satellite/ UC3MInstitucional/es/PortadaMiniSiteA/1371229065435/ 


\section{Apéndices}

\section{Apéndice A. Relación Producción Vs Precipitación}

Tabla 5 Relación Producción Vs Precipitación

\begin{tabular}{|c|c|c|}
\hline Año & Produccion & Precipitacion \\
\hline 1999-1 & $102,578 \mathrm{t}$ & $293.28 \mathrm{mms}$ \\
\hline $1999-2$ & $484,578 \mathrm{t}$ & $218.4 \mathrm{mms}$ \\
\hline $2000-1$ & $99,412 \mathrm{t}$ & $256.51 \mathrm{mms}$ \\
\hline $2000-2$ & $449,407 \mathrm{t}$ & $248.51 \mathrm{mms}$ \\
\hline $2001-1$ & $76,409 \mathrm{t}$ & $239.09 \mathrm{mms}$ \\
\hline $2001-2$ & $424,627 \mathrm{t}$ & $286.51 \mathrm{mms}$ \\
\hline $2002-1$ & $102,975 \mathrm{t}$ & $295.06 \mathrm{mms}$ \\
\hline $2002-2$ & $387,528 \mathrm{t}$ & $239.57 \mathrm{mms}$ \\
\hline 2003-1 & $79,283 \mathrm{t}$ & $244.97 \mathrm{mms}$ \\
\hline $2003-2$ & $466,038 \mathrm{t}$ & $261.44 \mathrm{mms}$ \\
\hline 2004-1 & $89,855 \mathrm{t}$ & $309.06 \mathrm{mms}$ \\
\hline 2004-2 & $460,311 \mathrm{t}$ & $269.29 \mathrm{mms}$ \\
\hline $2005-1$ & $89,428 \mathrm{t}$ & $278.34 \mathrm{mms}$ \\
\hline $2005-2$ & $378,146 \mathrm{t}$ & $234.38 \mathrm{mms}$ \\
\hline $2006-1$ & $61,819 \mathrm{t}$ & $269.97 \mathrm{mms}$ \\
\hline 2006-2 & $291,007 \mathrm{t}$ & $279.45 \mathrm{mms}$ \\
\hline $2007-1$ & $60,872 \mathrm{t}$ & $280.78 \mathrm{mms}$ \\
\hline $2007-2$ & $335,181 \mathrm{t}$ & $222.84 \mathrm{mms}$ \\
\hline 2008-1 & $86,487 \mathrm{t}$ & $205.87 \mathrm{mms}$ \\
\hline $2008-2$ & $415,349 \mathrm{t}$ & $278.02 \mathrm{mms}$ \\
\hline $2009-1$ & $62,695 \mathrm{t}$ & $260.32 \mathrm{mms}$ \\
\hline $2009-2$ & $504,863 \mathrm{t}$ & $238.12 \mathrm{mms}$ \\
\hline $2010-1$ & $42,503 \mathrm{t}$ & $289.08 \mathrm{mms}$ \\
\hline $2010-2$ & $447,577 \mathrm{t}$ & $260.15 \mathrm{mms}$ \\
\hline $2011-1$ & $85,081 \mathrm{t}$ & $275.7 \mathrm{mms}$ \\
\hline $2011-2$ & $306,385 \mathrm{t}$ & $250.21 \mathrm{mms}$ \\
\hline $2012-1$ & $50,960 \mathrm{t}$ & $255.12 \mathrm{mms}$ \\
\hline $2012-2$ & $275,707 \mathrm{t}$ & $256.83 \mathrm{mms}$ \\
\hline 2013-1 & $82,672 \mathrm{t}$ & $269.4 \mathrm{mms}$ \\
\hline 2013-2 & $224,181 \mathrm{t}$ & $294.79 \mathrm{mms}$ \\
\hline 2014-1 & $41,866 \mathrm{t}$ & $269 \mathrm{mms}$ \\
\hline 2014-2 & $150,769 \mathrm{t}$ & $262.31 \mathrm{mms}$ \\
\hline $2015-1$ & $49,252 \mathrm{t}$ & $262.68 \mathrm{mms}$ \\
\hline $2015-2$ & $234,395 \mathrm{t}$ & $239.46 \mathrm{mms}$ \\
\hline 2016-1 & $65,518 \mathrm{t}$ & $259.94 \mathrm{mms}$ \\
\hline $2016-2$ & $343,717 \mathrm{t}$ & $280.62 \mathrm{mms}$ \\
\hline $2017-1$ & $74,395 \mathrm{t}$ & $224.98 \mathrm{mms}$ \\
\hline $2017-2$ & $319,637 \mathrm{t}$ & $197.62 \mathrm{mms}$ \\
\hline
\end{tabular}

Fuente: Propia, elaborado a partir de datos del IDEAM y FEDEARROZ 


\section{Apéndice B. Análisis de Regresión Producción Vs Precipitación}

Tabla 6 Análisis de Regresión Producción Vs Precipitación

\begin{tabular}{|c|c|c|c|c|c|c|}
\hline & \multirow{2}{*}{\multicolumn{3}{|c|}{ Estadísticas de la regresión }} & & \\
\hline & & & & & & \\
\hline & & Coeficiente de & ción múltiple & 0.24349758 & & \\
\hline & & Coeficiente de & inación $\mathrm{R}^{\wedge} 2$ & 0.05929107 & & \\
\hline & & $\mathrm{R}^{\wedge} 2$ ajustado & & 0.03316027 & & \\
\hline & & Error típico & & 159898.164 & & \\
\hline & & Observaciones & & 38 & & \\
\hline & Coeficientes & Error típico & Estadístico $\mathrm{t}$ & Probabilidad & Inferior $95 \%$ & Superior $95 \%$ \\
\hline Intercepción & 621221.457 & 268601.202 & 2.31280222 & 0.02656099 & 76472.9704 & 1165969.94 \\
\hline Variable X 1 & -1552.39041 & 1030.58233 & -1.50632352 & 0.14070938 & -3642.50826 & 537.727438 \\
\hline
\end{tabular}

Tabla 7 Relación Producción Vs Precipitación-Área Sembrada

\begin{tabular}{|c|c|c|c|}
\hline Año & Produccion & Precipitacion & A. Sembrada \\
\hline 1999-1 & $102,578 \mathrm{t}$ & 293.28 & 87,204 ha \\
\hline $1999-2$ & $484,578 \mathrm{t}$ & 218.4 & 18,183 ha \\
\hline $2000-1$ & $99,412 \mathrm{t}$ & 256.51 & 84,787 ha \\
\hline $2000-2$ & $449,407 \mathrm{t}$ & 248.51 & 14,111 ha \\
\hline 2001-1 & $76,409 \mathrm{t}$ & 239.09 & 77,386 ha \\
\hline $2001-2$ & $424,627 \mathrm{t}$ & 286.51 & 18,239 ha \\
\hline $2002-1$ & $102,975 \mathrm{t}$ & 295.06 & 71,524 ha \\
\hline $2002-2$ & $387,528 \mathrm{t}$ & 239.57 & 13,981 ha \\
\hline $2003-1$ & $79,283 \mathrm{t}$ & 244.97 & 82,615 ha \\
\hline $2003-2$ & $466,038 \mathrm{t}$ & 261.44 & $17,003 \mathrm{ha}$ \\
\hline $2004-1$ & $89,855 \mathrm{t}$ & 309.06 & 83,848 ha \\
\hline $2004-2$ & $460,311 \mathrm{t}$ & 269.29 & 17,789 ha \\
\hline $2005-1$ & $89,428 \mathrm{t}$ & 278.34 & $71,025 \mathrm{ha}$ \\
\hline $2005-2$ & $378,146 \mathrm{t}$ & 234.38 & $11,401 \mathrm{ha}$ \\
\hline $2006-1$ & $61,819 \mathrm{t}$ & 269.97 & $53,051 \mathrm{ha}$ \\
\hline $2006-2$ & $291,007 \mathrm{t}$ & 279.45 & $10,723 \mathrm{ha}$ \\
\hline $2007-1$ & $60,872 \mathrm{t}$ & 280.78 & 59,249 ha \\
\hline $2007-2$ & $335,181 \mathrm{t}$ & 222.84 & 14,756 ha \\
\hline $2008-1$ & $86,487 \mathrm{t}$ & 205.87 & $72,257 \mathrm{ha}$ \\
\hline $2008-2$ & $415,349 \mathrm{t}$ & 278.02 & 11,546 ha \\
\hline $2009-1$ & $62,695 \mathrm{t}$ & 260.32 & 96,057 ha \\
\hline $2009-2$ & $504,863 \mathrm{t}$ & 238.12 & 7,894 ha \\
\hline $2010-1$ & $42,503 \mathrm{t}$ & 289.08 & 80,700 ha \\
\hline $2010-2$ & $447,577 \mathrm{t}$ & 260.15 & $16,481 \mathrm{ha}$ \\
\hline 2011-1 & $85,081 \mathrm{t}$ & 275.7 & 86,983 ha \\
\hline 2011-2 & $306,385 \mathrm{t}$ & 250.21 & $10,752 \mathrm{ha}$ \\
\hline 2012-1 & $50,960 \mathrm{t}$ & 255.12 & 56,719 ha \\
\hline $2012-2$ & $275,707 \mathrm{t}$ & 256.83 & $16,383 \mathrm{ha}$ \\
\hline 2013-1 & $82,672 \mathrm{t}$ & 269.4 & $61,640 \mathrm{ha}$ \\
\hline 2013-2 & $224,181 \mathrm{t}$ & 294.79 & 8,655 ha \\
\hline 2014-1 & $41,866 \mathrm{t}$ & 269 & 31,083 ha \\
\hline 2014-2 & $150,769 \mathrm{t}$ & 262.31 & 8,680 ha \\
\hline 2015-1 & $49,252 \mathrm{t}$ & 262.68 & $47,263 \mathrm{ha}$ \\
\hline $2015-2$ & $234,395 \mathrm{t}$ & 239.46 & $16,258 \mathrm{ha}$ \\
\hline 2016-1 & $65,518 \mathrm{t}$ & 259.94 & $63,312 \mathrm{ha}$ \\
\hline 2016-2 & $343,717 \mathrm{t}$ & 280.62 & $14,024 \mathrm{ha}$ \\
\hline 2017-1 & $74,395 \mathrm{t}$ & 224.98 & $68,052 \mathrm{ha}$ \\
\hline $2017-2$ & $319,637 \mathrm{t}$ & 197.62 & 14,705 ha \\
\hline
\end{tabular}

Fuente: Propia, elaborado a partir de datos del IDEAM y FEDEARROZ 


\section{Apéndice C. Relación Producción Vs Precipitación-Área Sembrada}

Tabla 8 Relación Producción Vs Precipitación-Área Sembrada

\begin{tabular}{|c|c|c|c|}
\hline Año & Produccion & Precipitacion & A. Sembrada \\
\hline $1999-1$ & $102,578 \mathrm{t}$ & 293.28 & 87,204 ha \\
\hline 1999-2 & $484,578 \mathrm{t}$ & 218.4 & 18,183 ha \\
\hline $2000-1$ & $99,412 \mathrm{t}$ & 256.51 & 84,787 ha \\
\hline $2000-2$ & $449,407 \mathrm{t}$ & 248.51 & 14,111 ha \\
\hline $2001-1$ & $76,409 \mathrm{t}$ & 239.09 & 77,386 ha \\
\hline $2001-2$ & $424,627 \mathrm{t}$ & 286.51 & 18,239 ha \\
\hline $2002-1$ & $102,975 \mathrm{t}$ & 295.06 & 71,524 ha \\
\hline $2002-2$ & $387,528 \mathrm{t}$ & 239.57 & 13,981 ha \\
\hline $2003-1$ & $79,283 \mathrm{t}$ & 244.97 & 82,615 ha \\
\hline 2003-2 & $466,038 \mathrm{t}$ & 261.44 & 17,003 ha \\
\hline 2004-1 & $89,855 \mathrm{t}$ & 309.06 & 83,848 ha \\
\hline 2004-2 & $460,311 \mathrm{t}$ & 269.29 & 17,789 ha \\
\hline 2005-1 & $89,428 \mathrm{t}$ & 278.34 & 71,025 ha \\
\hline $2005-2$ & $378,146 \mathrm{t}$ & 234.38 & 11,401 ha \\
\hline 2006-1 & $61,819 \mathrm{t}$ & 269.97 & 53,051 ha \\
\hline $2006-2$ & $291,007 \mathrm{t}$ & 279.45 & $10,723 \mathrm{ha}$ \\
\hline $2007-1$ & $60,872 \mathrm{t}$ & 280.78 & 59,249 ha \\
\hline $2007-2$ & $335,181 \mathrm{t}$ & 222.84 & 14,756 ha \\
\hline $2008-1$ & $86,487 \mathrm{t}$ & 205.87 & 72,257 ha \\
\hline $2008-2$ & $415,349 \mathrm{t}$ & 278.02 & 11,546 ha \\
\hline 2009-1 & $62,695 \mathrm{t}$ & 260.32 & 96,057 ha \\
\hline $2009-2$ & $504,863 \mathrm{t}$ & 238.12 & 7,894 ha \\
\hline $2010-1$ & $42,503 \mathrm{t}$ & 289.08 & 80,700 ha \\
\hline 2010-2 & $447,577 \mathrm{t}$ & 260.15 & 16,481 ha \\
\hline $2011-1$ & $85,081 \mathrm{t}$ & 275.7 & 86,983 ha \\
\hline 2011-2 & $306,385 \mathrm{t}$ & 250.21 & $10,752 \mathrm{ha}$ \\
\hline $2012-1$ & $50,960 \mathrm{t}$ & 255.12 & 56,719 ha \\
\hline $2012-2$ & $275,707 \mathrm{t}$ & 256.83 & $16,383 \mathrm{ha}$ \\
\hline 2013-1 & $82,672 \mathrm{t}$ & 269.4 & 61,640 ha \\
\hline 2013-2 & $224,181 \mathrm{t}$ & 294.79 & $8,655 \mathrm{ha}$ \\
\hline 2014-1 & $41,866 \mathrm{t}$ & 269 & $31,083 \mathrm{ha}$ \\
\hline 2014-2 & $150,769 \mathrm{t}$ & 262.31 & 8,680 ha \\
\hline 2015-1 & $49,252 \mathrm{t}$ & 262.68 & 47,263 ha \\
\hline 2015-2 & $234,395 \mathrm{t}$ & 239.46 & $16,258 \mathrm{ha}$ \\
\hline 2016-1 & $65,518 \mathrm{t}$ & 259.94 & 63,312 ha \\
\hline 2016-2 & $343,717 \mathrm{t}$ & 280.62 & 14,024 ha \\
\hline 2017-1 & $74,395 \mathrm{t}$ & 224.98 & 68,052 ha \\
\hline $2017-2$ & $319,637 \mathrm{t}$ & 197.62 & $14,705 \mathrm{ha}$ \\
\hline
\end{tabular}

Fuente: Propia, elaborado a partir de datos del IDEAM y FEDEARROZ 


\section{Apéndice D. Análisis de Regresión Producción Vs Precipitación-Área Sembrada}

Tabla 9 Análisis de Regresión Producción Vs Precipitación-Área Sembrada

\begin{tabular}{|c|c|c|c|c|c|c|}
\hline & \multirow{2}{*}{\multicolumn{3}{|c|}{ Estadísticas de la regresión }} & & \\
\hline & & & & & & \\
\hline & & \multicolumn{2}{|c|}{ Coeficiente de correlación múltiple } & 0.8077312 & & \\
\hline & & \multicolumn{2}{|c|}{ Coeficiente de determinación $\mathrm{R}^{\wedge} 2$} & 0.6524297 & & \\
\hline & & \multicolumn{2}{|l|}{$\mathrm{R}^{\wedge} 2$ ajustado } & 0.63256854 & & \\
\hline & & \multicolumn{2}{|l|}{ Error típico } & 98572.189 & & \\
\hline & & \multicolumn{2}{|l|}{ Observaciones } & 38 & & \\
\hline & Coeficientes & Error típico & Estadístico t & Probabilidad & Inferior $95 \%$ & Superior $95 \%$ \\
\hline Intercepción & 499969.471 & 166325.804 & 3.00596455 & 0.00487257 & 162310.138 & 837628.804 \\
\hline Variable X 1 & -410.385736 & 652.279526 & -0.62915624 & 0.53333208 & -1734.58357 & 913.812101 \\
\hline Variable X 2 & -4.16579001 & 0.53902211 & -7.72842142 & 4.4988E-09 & -5.26006306 & -3.07151695 \\
\hline
\end{tabular}

